\title{
Preferred Formation of Minority Concomitant Polymorphs in 2D Self- Assembly under Lateral Nanoconfinement
}

By

Johannes Seibel, [a] David B. Amabilino[b] and Steven De Feyter[a]*

[a] Dr. Johannes Seibel, Prof. Dr. Steven De Feyter

Department of Chemistry, Division of Molecular Imaging and

Photonics,

KU Leuven - University of Leuven

Celestijnenlaan 200F, B-3001 Leuven, Belgium

E-mail: steven.defeyter@kuleuven.be

[b] Prof. Dr. David B. Amabilino

School of Chemistry \& The GSK Carbon Neutral Laboratories for

Sustainable Chemistry

The University of Nottingham

Triumph Road, Nottingham NG7 2TU, UK

Abstract: Control over polymorph formation in the crystallization of organic molecules remains a huge scientific challenge. Here, we present preferential formation of one polymorph, formed by chiral molecules, in controlled two-dimensional (2D) nanoconfinement conditions at a liquid/solid interface. So-called nanocorrals to control concomitant polymorph formation were created in-situ via a nanoshaving protocol at the interface between 1-phenyloctane and covalently modified highly-oriented pyrolytic graphite (HOPG). The preferentially formed polymorphs - that were less stable in the largescale monolayers - could be selected simply by varying the orientation of the square nanocorrals with respect to the HOPG lattice. 
Polymorphism is the ability of a given compound to form at leasttwo different crystalline structures. While having the same chemical composition, each polymorph is unique with its own physical and chemical properties.[1] Due to these different properties, understanding and controlling polymorph formation of (chiral) organic compounds is of interest in pharmaceutical industries, $[2,3]$ organic electronics and functional materials in general. $[4,5]$ In particular in small-molecule organic semiconductors (OSCs), the crystal structure often plays an important role in device performance. Consequently, numerous strategies to control morphology and polymorphism of OSCs in thin films have been developed.[6] Among the methods available nanoscale confinement has been proven to be an efficient way to achieve control over polymorph formation,[7] for example by using well-defined pores having dimensions in the order of the critical nucleation size.[8] Lateral confinement in a line-pattern has been shown to increase the crystallinity and lead to the alignment of crystalline domains of a small-molecule organic semiconductor using capillary force lithography[9] and in thin films prepared by solution shearing and partial de-wetting.[10] In molecular self-assembly at surfaces and interfaces[11] twodimensional (2D) lateral nanoconfinement has been used to control the on-surface synthesis of organometallic chains and macrocycles.[12] Further, lateral nanoconfinement resulting from step-edges on atomically flat surfaces can affect polymorph formation of self-assembled molecular networks (SAMNs).[13,14]

However, these studies on lateral nanoconfinement of 2D SAMNs lack control over size and shape of the confinement conditions and the lateral confinement is static, as is the case in the OSCs confinement. In this context, we have developed an in-situ nanoshaving protocol at the liquid/solid interface, which uses scanning tunneling microscopy (STM) as manipulation and visualization tool.[15] This approach allows the control over size and shape of the nanoconfinement and, in contrast to the studies mentioned, the size and shape of the confinement changes gradually, potentially giving additional insights into kinetics and thermodynamics of the 2D crystallization process.

To achieve this, highly-oriented pyrolytic graphite (HOPG) is covalently modified, i.e. grafted, with 3,5-bis-tert-butylphenyl (3,5-TBP) moieties using diazonium chemistry. A high-density of grafted molecules prevents physisorption and SAMN formation from a drop-cast solution. The grafted molecules can be removed again to restore the pristine HOPG surface in the presence of the drop-cast solution, enabling SAMN formation in well-defined areas, so-called nanocorrals (Figure 1c).

Using this approach, we have previously shown that lateral confinement and the in-situ nanoshaving process can have a significant impact on SAMN formation in nanocorrals.[16-19] Here, we control nanocorral orientation to induce a bias in the formation of concomitant polymorphs, which differ in their orientation with respect to the HOPG lattice. Their preferential formation could be achieved simply by varying the orientation of square-shaped nanocorrals with respect to the HOPG lattice (Figure 1). Further, we show how hydrogen bond (H-bond) directionality in combination with the insitu nanoshaving process affects the molecular orientation and selection efficiency in the nanocorrals.

To achieve polymorph selection we chose molecules with a high aspect ratio, forming polymorphous structures having different angles with respect to the underlying HOPG lattice. Upon dropcasting a $10 \mathrm{mM}$ solution of enantiopure 4-[(S/R-1- methylheptyl)oxy]4'-biphenylformamide (S/R-BFA)[20] in 1- phenyloctane (PO) onto pristine HOPG, the molecules form an SAMN, appearing as a row-like structure in large-scale STM images (see supporting information S1 for experimental details) with two different orientations, taking into account the threefold symmetry of the surface (Figure 2a). 
High-resolution STM images reveal that these rows consist of head-to-tail dimers (Figure 2b). Imaging of the underlying HOPG lattice showed that the two different orientations have the same unit cell dimensions and only differ discernibly in their orientation with respect to the HOPG lattice (Figure $2 \mathrm{c}$ ). While we focus on one enantiomer in the following description of the obtained results, measurements were carried out with both enantiomers. The angles between the row-like features of the enantiomers and main symmetry axes of the HOPG lattice have opposite signs, i.e. $+9^{\circ}(\theta 1)$ and $+24^{\circ}(\theta 2)$ for R-BFA and $-9^{\circ}$ and $-24^{\circ}$ for S-BFA, respectively (Figure S1).

Square-shaped nanocorrals with the fast-shaving direction aligned along the long molecular axis of one of the polymorph structures, were used in the selection experiments. The row-like features, which are parallel to the short unit cell vector a and orthogonal to the long molecular axis, run parallel to the slow-shaving direction (Figure 2 ). Thereby, a bias in polymorph formation could be achieved simply by adjusting the nanocorral orientation (Figure 2e): Induction of the $\pm 24^{\circ}$ structure was more selective, which is the least favored polymorph in full monolayers on pristine HOPG (Figure S2). Note that while figures $2 \mathrm{~d}$ and $3 \mathrm{~d}$ are composed of two STM images for better illustration, the measurements were actually carried out with sets of four squares of the same orientation to minimize drift effects (Figure S3). In figure 2e the statistics are summarized for both enantiomers combined. More detailed statistics for each enantiomer are in the supporting information (Figure S4).

Rotational domains were rarely observed and all corrals contained only a single domain. The second molecule studied, enantiopure 4-[(S/R-1-methylheptyl)oxy]phenyl-4'formamidobenzoate (S/R-PFB, figure $1 \mathrm{~b}),[20,21]$ also forms row-like structures upon drop-casting a $5.5 \mathrm{mM}$ solution in PO onto pristine HOPG with two different orientations (Figure $3 \mathrm{a}$ and $\mathrm{b}$ ). These rows either run in $\mathrm{a} \pm 30^{\circ}$ angle $(\Theta 2)$ with respect to a highsymmetry surface direction or are tilted with an angle $(\Theta 1)$ of $-22^{\circ}$ (R-PFB) or $+22^{\circ}$ (S$\mathrm{PFB}$ ), respectively (Figure $3 \mathrm{c}$ and $\mathrm{S} 1$ ). Interestingly, adjacent polymorphs can merge without showing a clear domain boundary (Figure $3 \mathrm{~b}$ ). Similar to the BFA system, we first attempted the polymorph selection by creating squares with the long molecular axis aligned along the fast-shaving direction.

However, this orientation did not result in any alignment of the rows parallel to the nanocorral side border (Figure S5). Thus, we attempted the selection of the polymorph with the short unit cell vector parallel to the top border (Figure 3d). Using this square orientation we were able to achieve polymorph selection, which showed a surprisingly high efficiency considering the difference of only $8^{\circ}$ between the two polymorphs (Figure $3 e$ ). The statistics shown in figure $3 e$ are summarized from measurement sessions with both enantiomers. Detailed statistics are shown in the supporting information (Figure S6). More than one domain in a corral was rarely observed, but some corrals were only partially filled (Figure S7). Notably, the highest selection was achieved for the \pm 22 polymorph of the PFB system, which is the minority polymorph in the SAMN on pristine HOPG (Figure S2).

Apart from the polymorph selection efficiency, the main difference between the two systems is the orientation of the row structure and long molecular axis in the selected domains. In the case of BFA, the long molecular axis is aligned along the fast-shaving direction and in contrast thereto, the PFB long molecular axis is aligned along the slowshaving direction (Figure 4). Previously, we proposed that geometric constraints due to the long aspect ratio of the molecules in the initial stages of nanocorral formation play a major role in the observed domain selection.[16-18,22] This would, however, only explain the observed polymorph selection of the R/S-BFA system and is clearly not the 
case for the R/S-PFB system. This can be understood by looking at the directionality of possible intermolecular interactions in the confined 2D crystal structures (Figure 4).

While BFA and PFB can both potentially form $\mathrm{H}$-bonds via their formamide head-groups, the directionality of the intermolecular $\mathrm{H}$-bonds in relation to the long molecular axis is significantly different. The BFA structure consists of head-to-tail dimers, which align in a straight line perpendicular to the long molecular axis (Figure 4a). This alignment allows $\mathrm{H}$-bonding between the dimers, i.e. between adjacent rows, and van-der-Waals (vdW) interactions along the row structure. In contrast thereto, PFB assembles in rows of single molecules, resulting in intermolecular $\mathrm{H}$-bonds and $\mathrm{vdW}$ interactions along the row structure (Figure $4 \mathrm{~b}$ ). Thus, in both molecular systems the intermolecular interactions are anisotropic and the $\mathrm{H}$-bond direction is parallel to the fast shaving direction in the preferentially formed polymorphs.

Due to the formation of single domains even in nanocorrals as large as $220 \mathrm{~nm} \times 220$ $\mathrm{nm}$ (Figure S8), we propose nucleation as the deciding step, i.e. the first polymorph that nucleates is the one growing as the nanocorral is being formed. Supporting this claim, we observed that ex-situ nanocorrals (created before drop-casting the solution) showed SAMNs formation with multiple domains and no alignment effects (Figure S9), highlighting the impact of the nanoshaving process on molecular self-assembly. That is, in the initial stages of nanocorral formation, when nucleation is expected to occur, the area available for molecular self-assembly has a rectangular shape and high aspect ratio, imposing geometrical constraints on the nucleus shape and thus stability, depending on the nucleus' orientation with respect to the nanocorral (Figure 4). Additionally, interactions between the SAMN and the nanocorral edges may affect the nucleus stability. A small high aspect ratio BFA nucleus of several adjacent short rows is thermodynamically favored due to a higher number of $\mathrm{H}$-bonds, compared to fewer long rows (Figure S5 and S10) and the other polymorph (Figure 4a). Therefore, the former will be the first one to reach a critical stability on the surface under the given constraints. In contrast thereto, the intermolecular interactions in the PFB system are optimized by the formation of long rows rather than by several adjacent shorter rows (Figure S11). These long rows, however, can only form when they run exactly parallel to the top border of the nanocorral (Figure 4b). As a result, the selectivity in the PFB system is higher even though the angle between the two polymorphs is smaller compared to the BFA system. This anisotropy is also reflected in the assembly of BFA in narrow rectangular nanocorrals (Figure S12). That is, BFA assembles in a narrow rectangular nanocorral with its long axis parallel to the fast shaving, i.e. H-bond direction, while a nanocorral of the same size, but the long axis parallel to the slow shaving direction remains empty. Furthermore, the higher selectivity in the nanocorrals of the minority polymorph suggests that it is kinetically preferred and may have a smaller critical nucleation size, while the other one is thermodynamically more stable. A further decrease in the relative appearance of the minority polymorphs on pristine HOPG after annealing supports this hypothesis (Figure S13).

In summary, we have presented preferential polymorph formation by enantiopure molecules at the liquid/solid interface using in-situ created nanocorrals. The polymorph selection solely depended on the orientation of the nanocorral with respect to the HOPG lattice, which can be chosen freely employing the nanoshaving protocol presented here. Anisotropic intermolecular interactions in the assembled structures are proposed to play a major role in the selection mechanism. We believe the domain selection with in-situ nanoshaving to be general phenomena in molecular systems with strong anisotropic intermolecular interactions that quickly nucleate, making this method a valuable tool to study molecular crystallization and nucleation at surfaces and interfaces. The potential of the method is proven by the fact that polymorphs that are the minority in large scale 
monolayers can be favoured under nanoconfinement. Future work will involve racemic mixtures of the compounds studied here and their enantioselective adsorption in nanocorrals.

\section{Acknowledgements}

The authors gratefully acknowledge financial support from the Fund of Scientific Research Flanders (FWO), KU Leuven - Internal Funds, and FWO under EOS 30489208. J. S. acknowledges financial support through a Marie Skłodowska-Curie Individual Fellowship (EU project 789865 - EnSurf). D. B. A. thanks the EPSRC (project $\mathrm{EP} / \mathrm{M005178/1)}$ and the School of Chemistry at the University of Nottingham for funding.

\section{REFERENCES}

[1] J. Bernstein, R. J. Davey, J.-O. Henck, Angew. Chemie Int. Ed. 1999, 38, 34403461.

[2] Z. Gao, S. Rohani, J. Gong, J. Wang, Engineering 2017, 3, 343-353.

[3] A. Llinàs, J. M. Goodman, Drug Discov. Today 2008, 13, 198-210.

[4] D. B. Amabilino, D. K. Smith, J. W. Steed, Chem. Soc. Rev. 2017, 46, 2404-2420.

[5] D. Gentili, M. Gazzano, M. Melucci, D. Jones, M. Cavallini, Chem. Soc. Rev 2019, 48, 2502.

[6] Y. Diao, L. Shaw, Z. Bao, S. C. B. Mannsfeld, Energy Environ. Sci. 2014, 7, 21452159.

[7] Q. Jiang, M. D. Ward, Chem. Soc. Rev. 2014, 43, 2066-2079.

[8] J. M. Ha, J. H. Wolf, M. A. Hillmyer, M. D. Ward, J. Am. Chem. Soc. 2004, 126, 3382-3383.

[9] H. Kwon, K. Kim, T. K. An, S. H. Kim, C. E. Park, J. Ind. Eng. Chem. 2019, 75, 187193.

[10] G. Giri, S. Park, M. Vosgueritchian, M. M. Shulaker, Z. Bao, Adv. Mater. 2014, 26, 487-493.

[11] R. Raval, Faraday Discuss. 2017, 204, 9-33.

[12] Q. Fan, J. Dai, T. Wang, J. Kuttner, G. Hilt, J. M. Gottfried, J. Zhu, ACS Nano 2016, $10,3747-3754$.

[13] D. Kühne, F. Klappenberger, R. Decker, U. Schlickum, H. Brune, S. Klyatskaya, M. Ruben, J. V Barth, J. Phys. Chem. C 2009, 113, 17851-17859.

[14] D. G. De Oteyza, E. Barrena, H. Dosch, Y. Wakayama, Phys. Chem. Chem. Phys. $2009,11,8741-8744$.

[15] J. Greenwood, T. H. Phan, Y. Fujita, Z. Li, O. Ivasenko, W. Vanderlinden, H. Van Gorp, W. Frederickx, G. Lu, K. Tahara, et al., ACS Nano 2015, 9, 5520-5535.

[16] L. Verstraete, J. Greenwood, B. E. Hirsch, S. De Feyter, ACS Nano 2016, 10, 10706-10715. 
[17] J. Seibel, L. Verstraete, B. E. Hirsch, A. M. Braganca, S. De Feyter, J. Am. Chem. Soc. 2018, 140, 11565-11568.

[18] L. Verstraete, J. Smart, B. E. Hirsch, S. De Feyter, Phys. Chem. Chem. Phys. 2018, 20, 27482-27489.

[19] Y. Hu, A. M. Bragança, L. Verstraete, O. Ivasenko, B. E. Hirsch, K. Tahara, Y. Tobe, S. De Feyter, Chem. Commun. 2019, 55, 2226-2229.

[20] W. Mamdouh, H. Uji-i, A. Gesquière, S. De Feyter, D. B. Amabilino, M. M. S. AbdelMottaleb, J. Veciana, F. C. De Schryver, Langmuir 2004, 20, 9628-9635.

[21] S. De Feyter, A. Gesquire, K. Wurst, D. B. Amabilino, J. Veciana, F.C. De Schryver, Angew. Chemie - Int. Ed. 2001, 40, 3217-3220.

[22] L. Verstraete, B. E. Hirsch, J. Greenwood, S. De Feyter, Chem. Commun. 2017, 53, 4207-4210. 

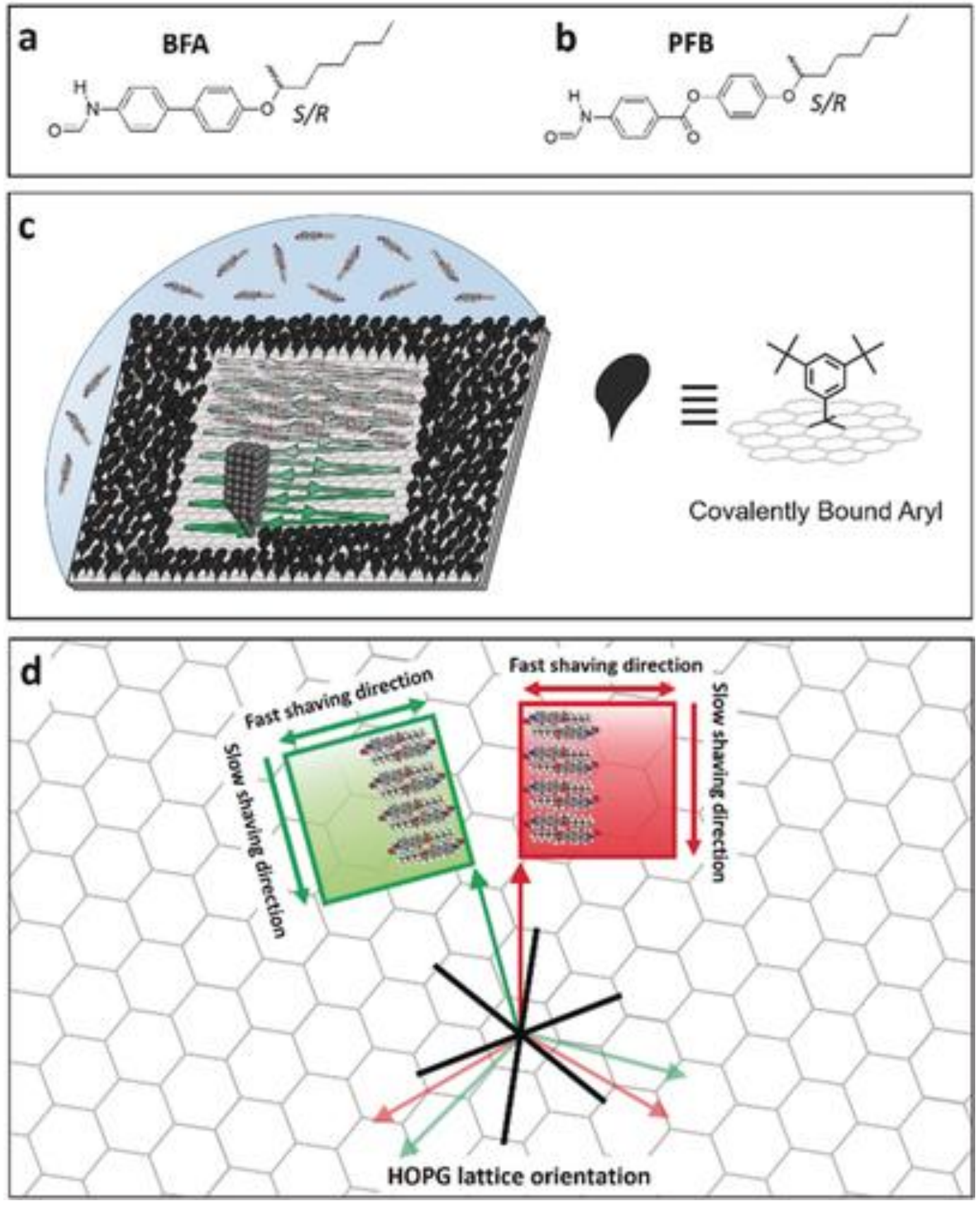

Figure 1. Structures of the molecules studied ( $a$ and $b$ ) and schematic representation of the in-situ nanoshaving process used to create nanocorrals for polymorph selection (c). Panel $d$ shows the principle of polymorph selection using square-shaped nanocorrals with different orientations with respect to the HOPG lattice (not to scale). Rotational domains are indicated by arrows. 

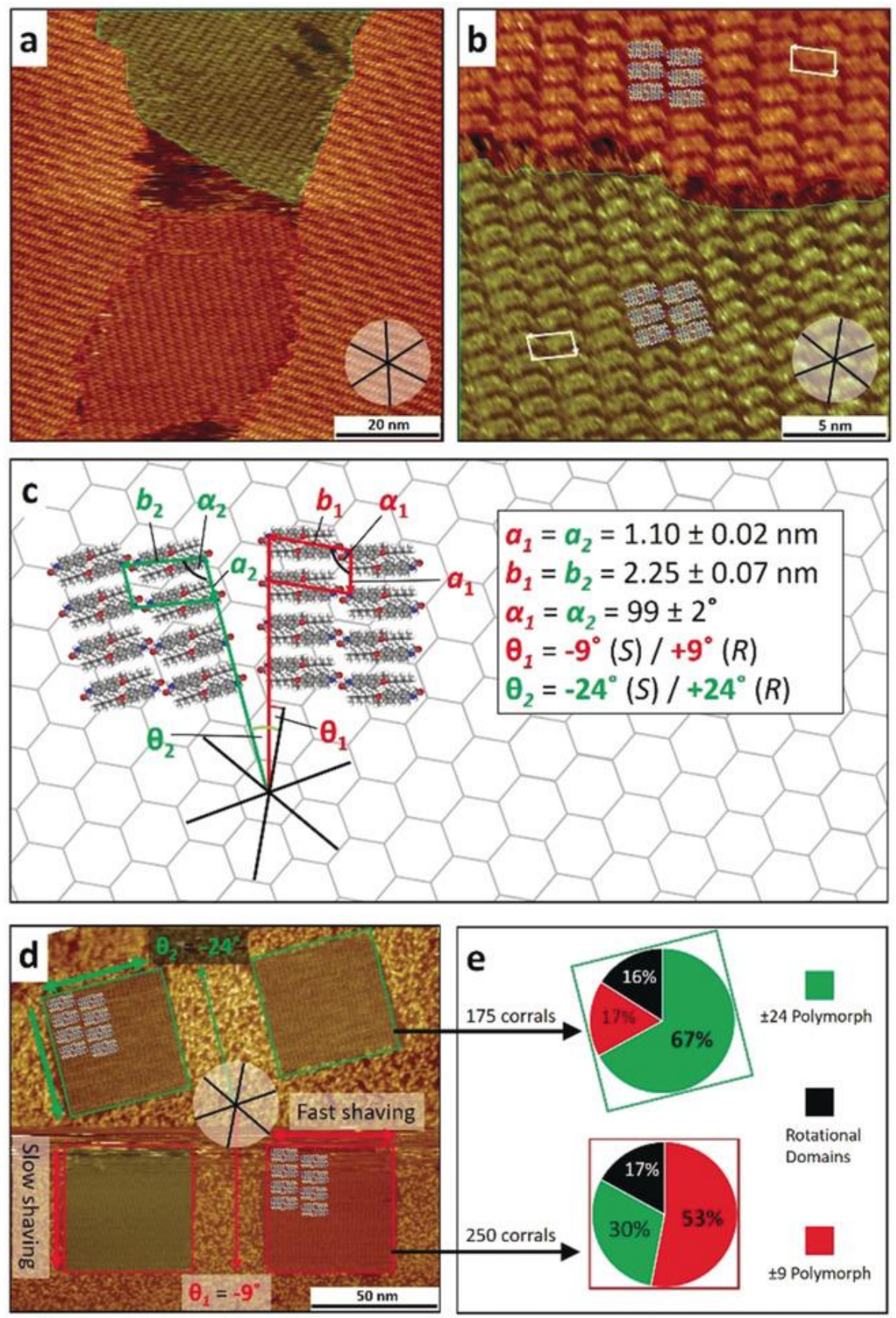

Figure 2. Large scale (a) and high-resolution (b) STM images of S-BFA at the pristine PO/HOPG interface at a concentration of $10 \mathrm{mM}$. Different polymorphs are colored red and green, respectively. The unit cell and molecular assembly for each polymorph is indicated in b. Panel c shows a tentative model for the assembly of S-BFA in relation to the HOPG lattice and the unit cell parameters for both enantiomers. In-situ created nanocorrals with angles of $-9^{\circ}$ (bottom) and $-24^{\circ}$ (top) with respect to the HOPG lattice are shown in $\mathrm{d}$. Molecular models indicate the assembly in relation to the nanocorral. Statistics of the polymorph distribution in the nanocorrals (e) show a clear bias towards the polymorph with the short unit cell vector parallel to the slow shaving direction. The black bar represents other rotational domains. Imaging parameters: It $=70 \mathrm{pA}, \mathrm{Vb}=-$ $0.700 \mathrm{~V}$. 

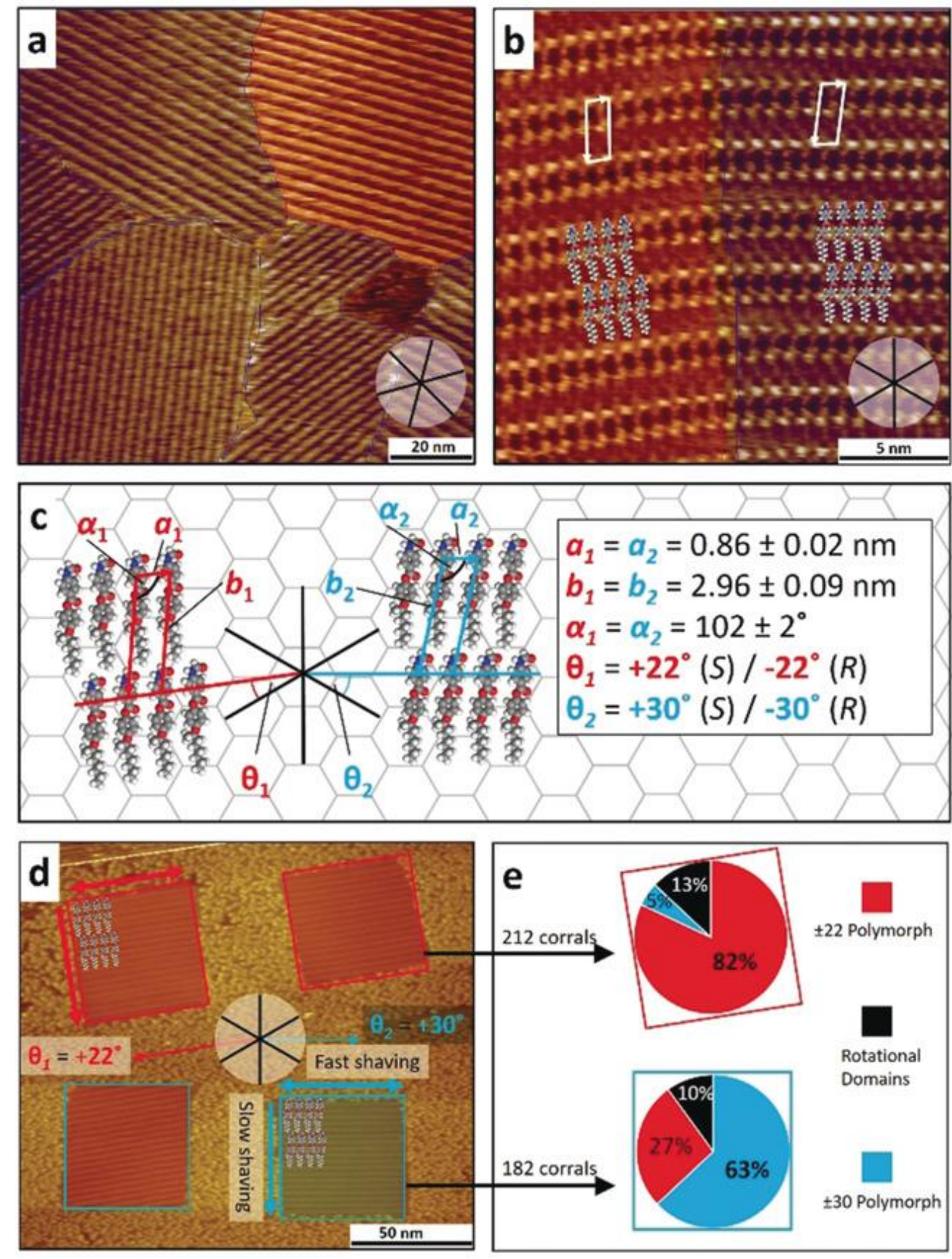

Figure 3. Large scale (a) and high-resolution (b) STM images of S-PFB at the pristine $\mathrm{PO} / \mathrm{HOPG}$ interface at a concentration of $5.5 \mathrm{mM}$. Different polymorphs are colored red and blue. The unit cell and molecular assembly for each polymorph is indicated in $b$. Panel c shows a tentative model of the assembly of S-PFB in relation to the HOPG lattice and the unit cell parameters for both enantiomers. In-situ created nanocorrals with angles of $+22^{\circ}$ (top) and $+30^{\circ}$ (bottom) with respect to the HOPG lattice are shown in $d$. Molecular models indicate the assembly in relation to the nanocorral. Statistics of the polymorph distribution in the nanocorrals (e) show a clear bias towards the polymorph with the row-structure parallel to the fast shaving direction. The black bar represents other rotational domains. Imaging parameters: It $=70 \mathrm{pA}, \mathrm{Vb}=-0.700 \mathrm{~V}$. 

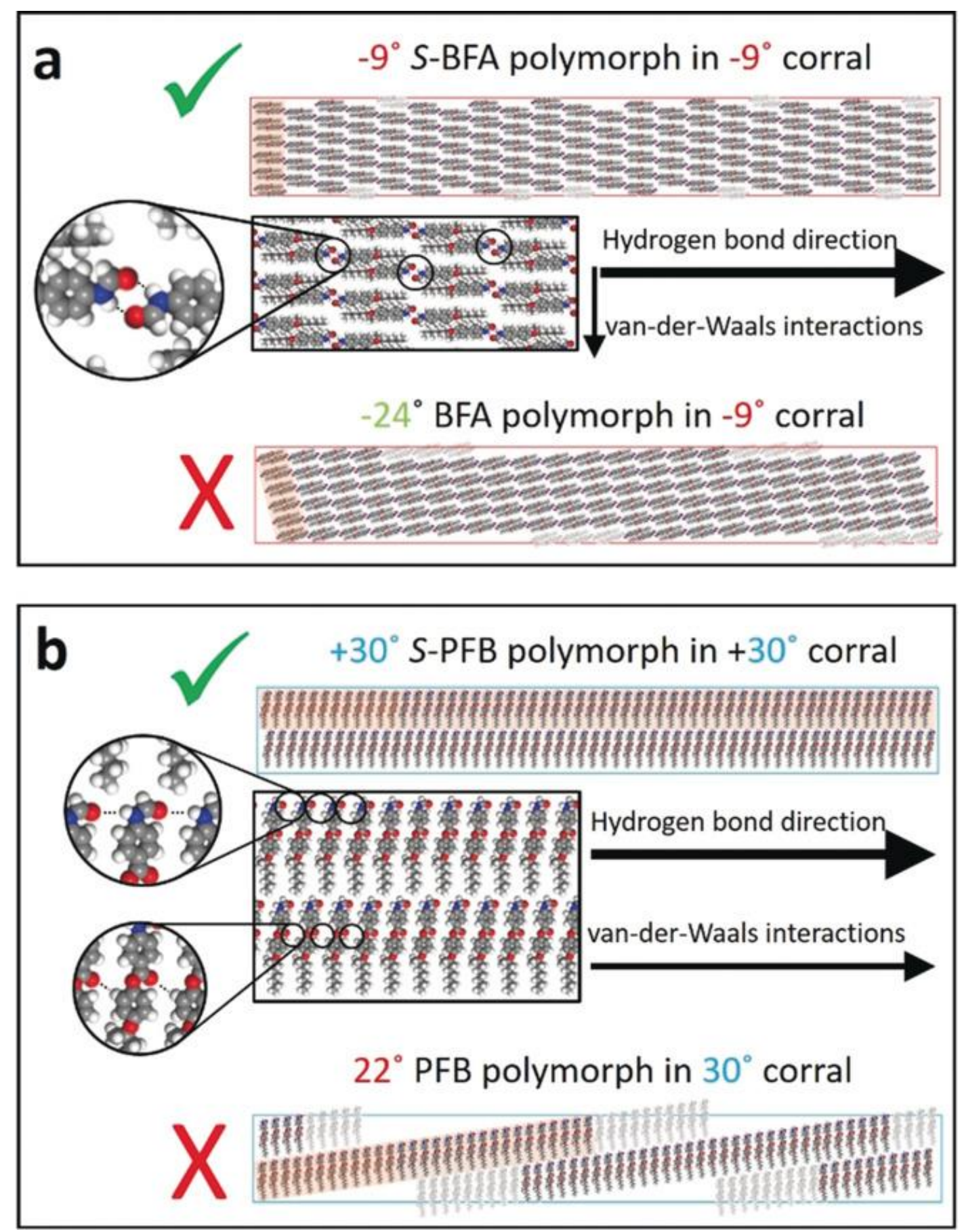

Figure 4. Tentative models of the molecular orientation of both polymorphs of BFA (a) and PFB (b), respectively, in the same nanocorral. The rectangular shape of the nanocorral is chosen to represent the shape during the initial stages of the nanoshaving process. Both of the preferentially-formed polymorphs have $\mathrm{H}$-bond directionality along the fast-shaving direction. Additional vdW interactions are possible perpendicular to the $\mathrm{H}$-bonds along the slow shaving direction in the BFA system and parallel to the $\mathrm{H}$-bonds in the PFB system. In the case of BFA, the mismatching polymorph contains slightly less molecules and thus $\mathrm{H}$-bonds (119 vs 113 dimers), while the difference between matching and mismatching polymorph of PFB is significantly higher (115 vs 75 molecules). 


\section{Supporting Information}

Preferred Formation of Minority Concomitant Polymorphs in 2D Self-Assembly under Nanoconfinement

Johannes Seibel ${ }^{1}$, David B. Amabilino ${ }^{2}$ and Steven De Feyter ${ }^{1}$

' Division of Molecular Imaging and Photonics, Department of Chemistry, KU Leuven, Celestijnenlaan 200F, 3001 Leuven, Belgium

${ }^{2}$ School of Chemistry \& The GSK Carbon Neutral Laboratories for Sustainable Chemistry, The University of Nottingham, Triumph Road, Nottingham NG7-2TU, UK

Corresponding author: steven.defeyter@kuleuven.be

\section{Contents:}

S1. Experimental details ............................................................................... $\mathrm{S} 2$

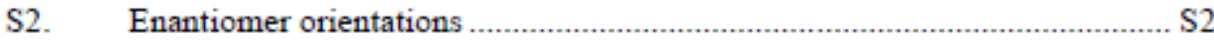

S3. Polymorph distribution of BFA and PFB on pristine HOPG ..................... S3

S4. Nanocorral alignment in the measurements ................................................ S4

S5. Detailed statistics of BFA polymorph selection ......................................... S5

S6. PFB parallel polymorph selection attempt ................................................... S6

S7. Detailed statistics of PFB polymorph selection …...................................... S7

S8. Partially filled nanocorrals ...................................................................... S8

S9. Single domains in larger nanocorrals ..................................................... S9

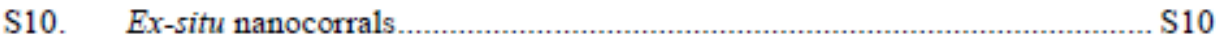

S11. Molecular assembly of BFA in $90^{\circ}$ rotated nanocorrals ........................... S11

S12. Molecular assembly of PFB in $90^{\circ}$ rotated nanocorrals ............................. S12

S13. Narrow rectangular nanocorrals .............................................................. 113

S14. Polymorph distribution after annealing................................................ S14 


\section{S1. Experimental details}

STM measurements were performed with a Agilent PicoLE system in constant-current mode (PicoSPM) at the 1-phenyloctane/highly-oriented pyrolytic graphite (PO/HOPG) interface at room temperature $\left(20-22{ }^{\circ} \mathrm{C}\right)$. The imaging parameters $I_{t}$ (tunneling current) and $V_{b}$ (bias applied to the sample) are indicated in the figure captions. Nanoshaving experiments were performed using PicoLITH v.2.1 software. STM tips were obtained by mechanical cutting of a Pt/lr wire $(80 \% / 20 \%$, diameter $0.25 \mathrm{~mm})$. Immediately before use, the HOPG substrate (grade ZYB, Advanced Ceramics Inc., Cleveland, OH, USA) was freshly cleaved using adhesive tape. The solvent, 1-phenyloctane (Sigma-Aldrich, 98\%), was used without further purification. Scanning Probe Imaging Processor software (SPIP 6.5.1) from Image Metrology ApS was used for STM image processing.

\section{S2. Enantiomer orientations}
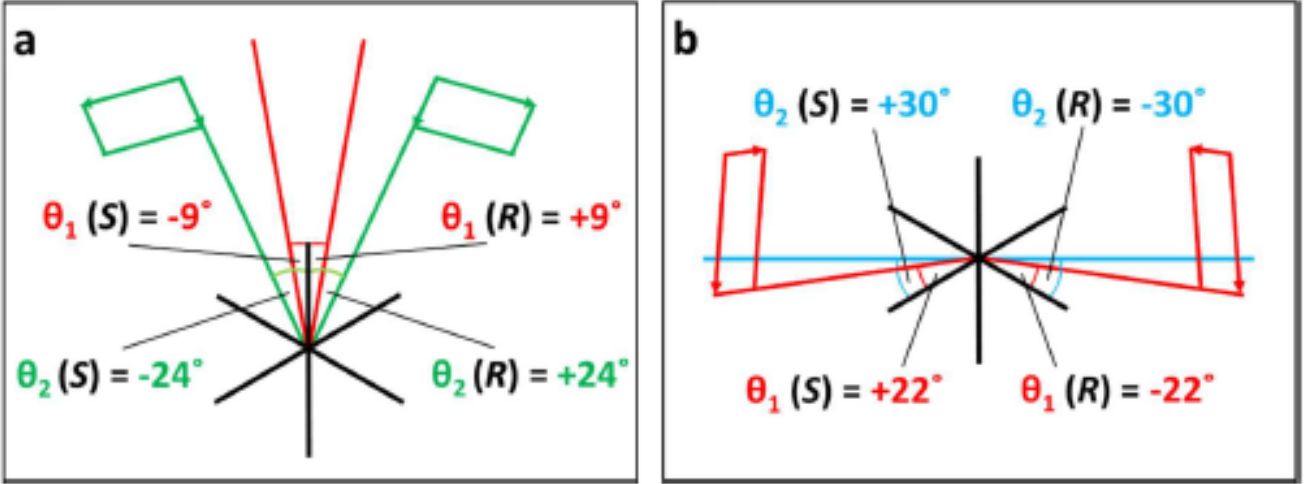

Figure S1. Schematic showing the opposite angles for the enantiomers of BFA (a) and PFB (b), respectively, with respect to the underlying HOPG lattice. The closest angle to the left of an HOPG symmetry direction is defined as ${ }^{\prime \prime}$ ", while angles to the right are ${ }^{\prime \prime}{ }^{\prime \prime}$. 
S3. Polymorph distribution of BFA and PFB on pristine HOPG
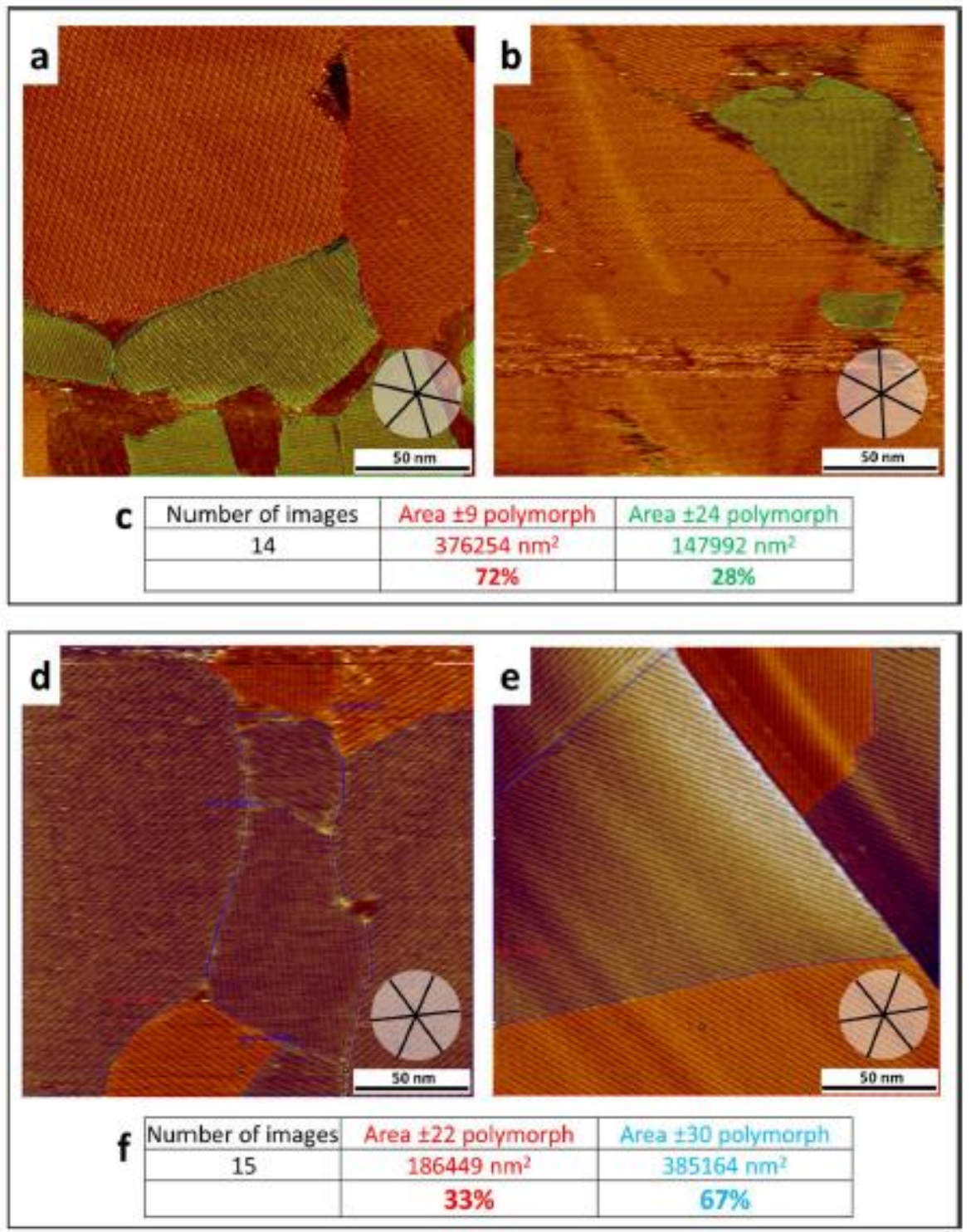

Figure S2. Representative STM images of S-BFA on pristine HOPG ( $\mathrm{a}$ and $\mathrm{b}$ ) and R-PFB ( $\mathrm{d}$ and e) used to determine the polymorph distribution. Therefore, the area covered by each polymorph was measured on several STM images. Statistics from $14200 \mathrm{~nm} \times 200 \mathrm{~nm}$ images showed that the \pm 24 BFA polymorph covered an area of only $28 \%$, while $72 \%$ are covered by the \pm 9 BFA polymorph (c). Similarly, statistics from $15200 \mathrm{~nm} \times 200 \mathrm{~nm}$ images showed that the \pm 22 PFB polymorph covered an area of only $33 \%$, while $67 \%$ are covered by the $\pm 30 \mathrm{PFB}$ polymorph (f). Imaging parameters: $I_{t}=70 \mathrm{pA}, V_{b}=-0.700 \mathrm{~V}$. 
S4. Nanocorral alignment in the measurements
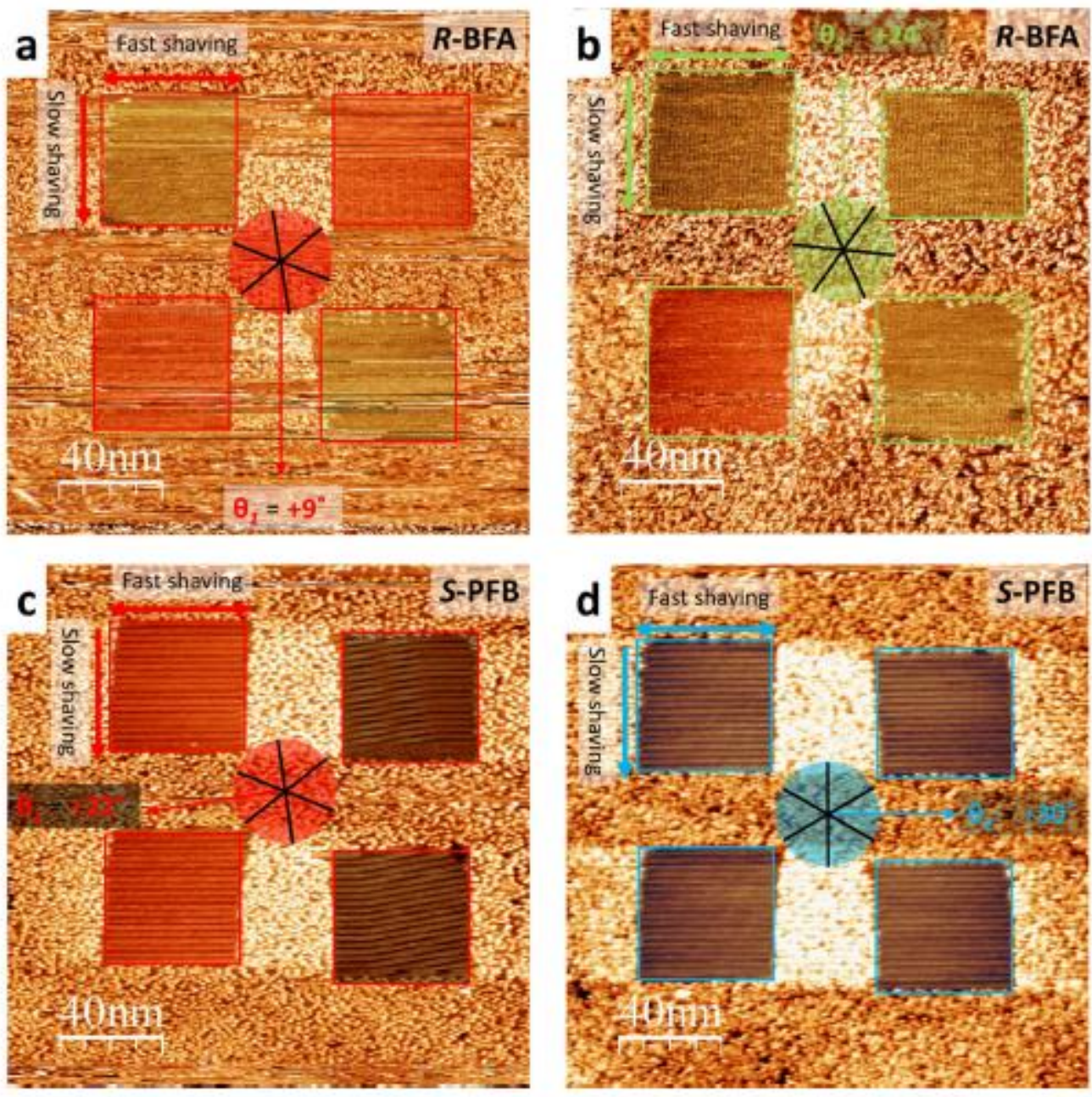

Figure S3. Representative STM images showing the nanocorral alignment in the performed measurements. To reduce the effect of drift, four nanocorrals with the fast shaving direction parallel to the fast scanning direction were created. Different polymorphs were selected by rotating the scanning direction with respect to the HOPG lattice. Thus, a shows nanocorrals for the selection of the $+9^{\circ} R-B F A$ and $b$ the $+24^{\prime}$ polymorph, respectively. In a the $+22^{\circ}$ polymorph of S-PFB is selected and in $d$ the $+30^{\circ}$ polymorph. The two squares on the right in a contain two different polymorphs, which was occasionally observed. These nanocorrals are denoted as "multiple domains" in the detailed statistics in figure S5, but were not included in the polymorph distribution statistics. Imaging parameters: $l_{t}=70 \mathrm{pA}, V_{b}=-$ $0.700 \mathrm{~V}$. 
S5. Detailed statistics of BFA polymorph selection
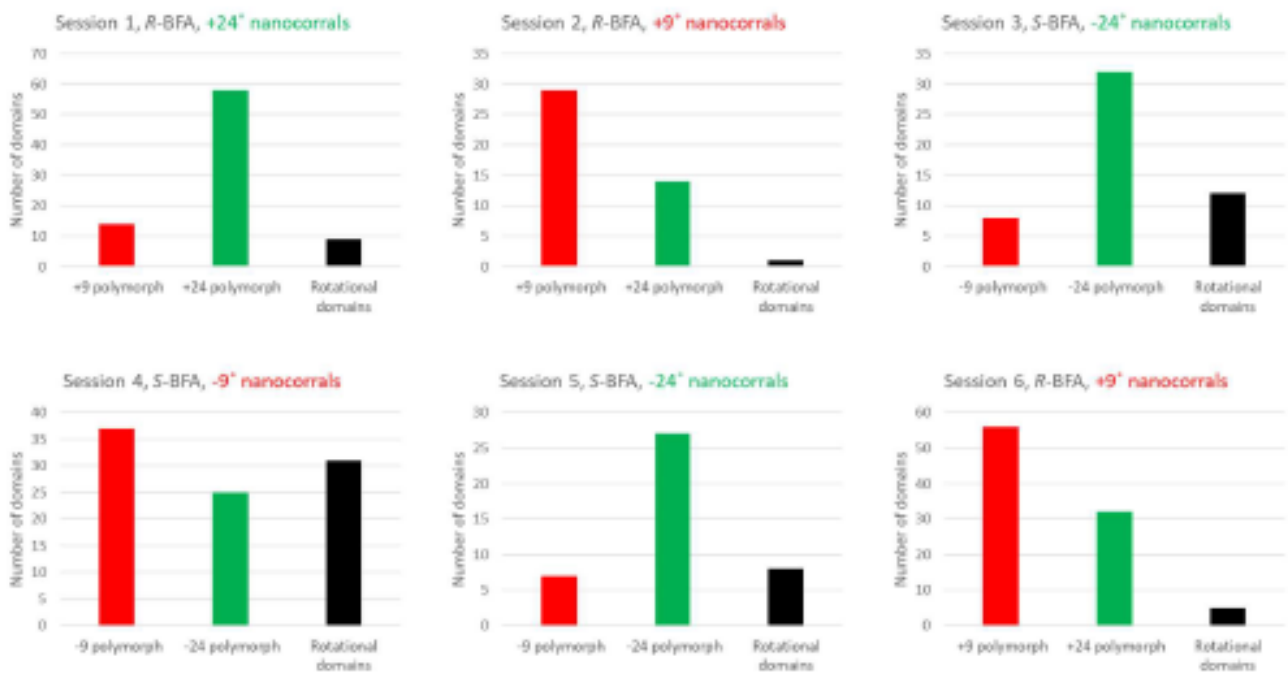

Figure S4. Statistics of the polymorph distribution in each measurement session, i.e. measurements which were performed consecutively with the same sample and STM tip. While there are small differences in the polymorph distribution between sessions, the general trend is the same, albeit with session 4 showing a significant higher number of rotational domains. 


\section{S6. PFB parallel polymorph selection attempt}
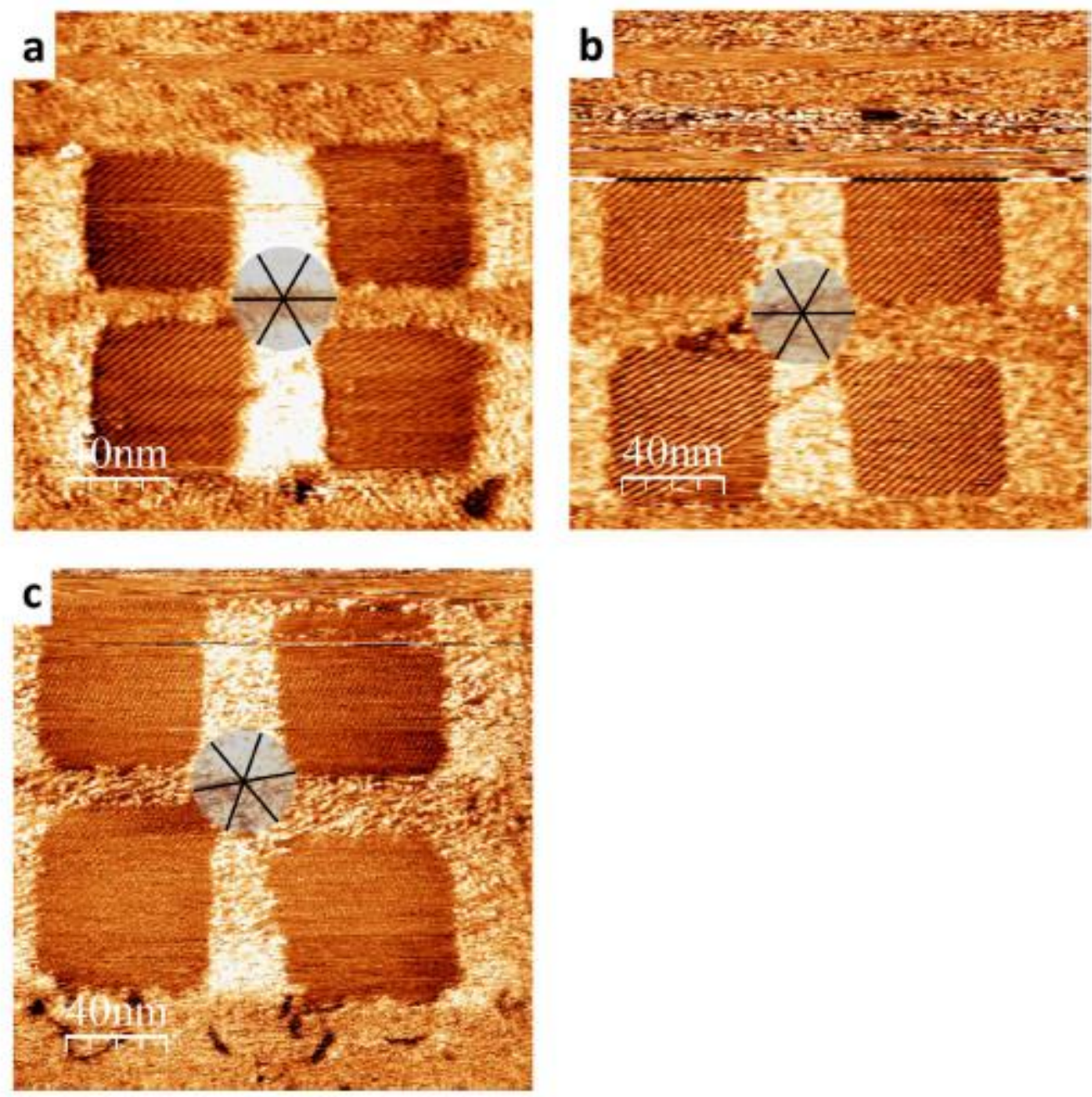

Figure S5. Representative STM images showing S-PFB in square nanocorrals oriented to select the polymorph with the row structure running parallel to the slow shaving direction, i.e. from top to bottom. However, with this nanocorral orientation only diagonal domains were observed without any bias in the polymorph formation. The STM image in c has the fast shaving direction rotated by 90 degree compared to the nanocorrals used for successful BFA polymorph selection, showing the formation of rotational $R$ BFA domains rather than the domain with the row structure parallel to the fast shaving direction. Imaging parameters: $I_{t}=70 \mathrm{pA}, V_{0}=-0.700 \mathrm{~V}$. 
S7. Detailed statistics of PFB polymorph selection
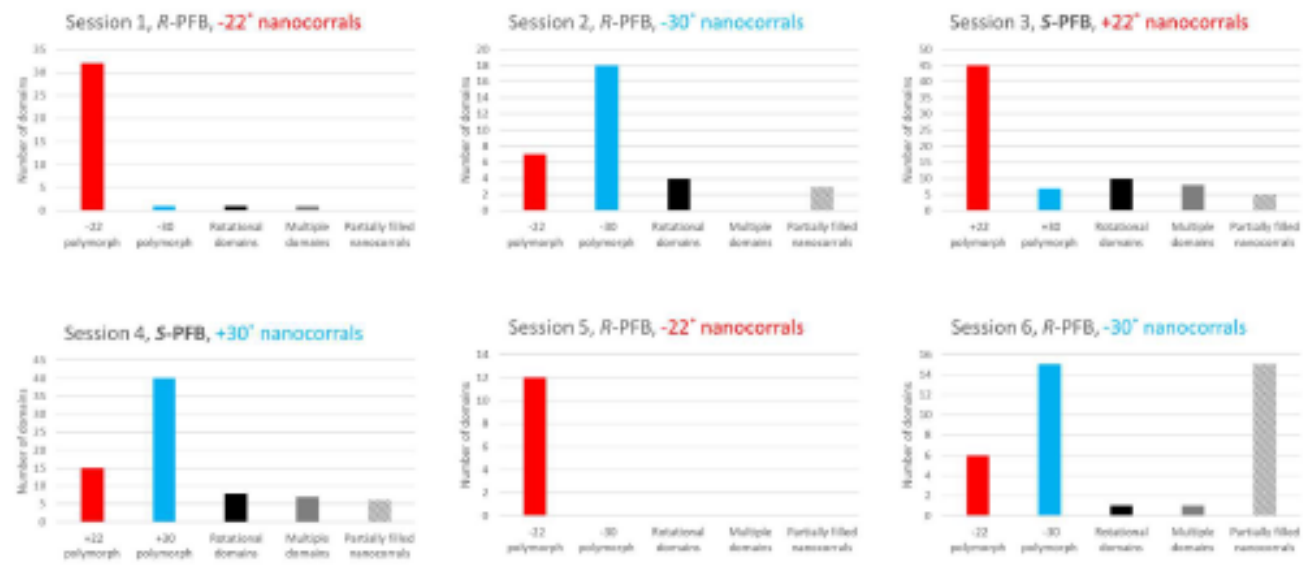

Session 7, R-PFB, -22* nanocorrals
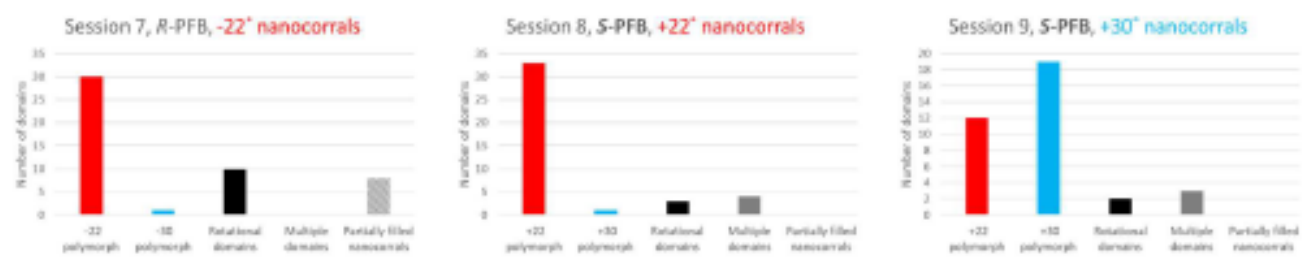

Figure S6. Statistics of the polymorph distribution in each measurement session, i.e. measurements which were performed consecutively with the same sample and STM tip. "Multiple domains" are nanocorrals filled with more than one polymorph (in most cases two, see figure S3c) and partially filled nanocorrals are nanocorrals with empty areas (Figure S7). While there are small differences in the polymorph distribution between sessions, the general trend between sessions and for both enantiomers is the same. 
S8. Partially filled nanocorrals
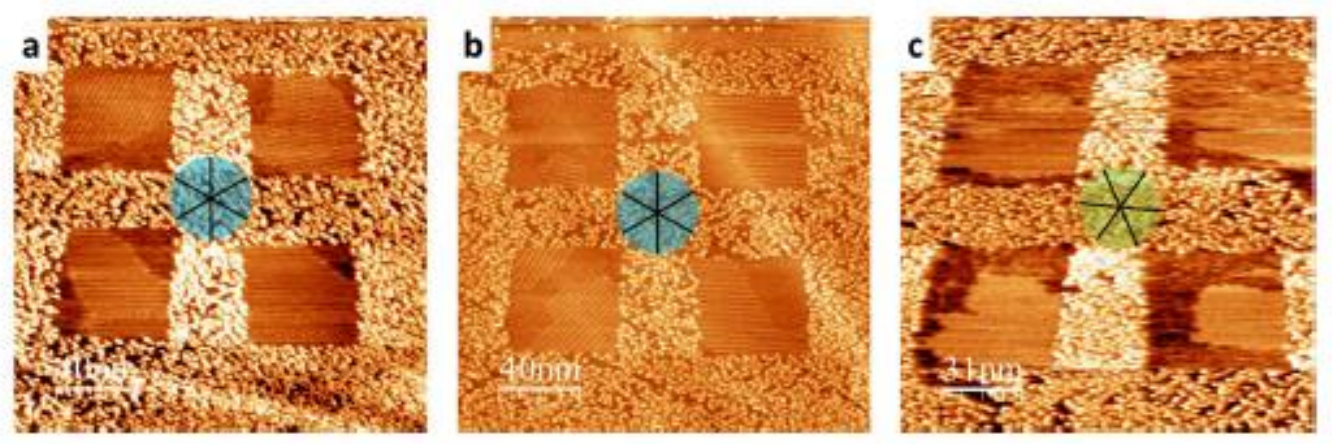

Figure S7. Representative STM images showing partially filled nanocorrals, which was occasionally observed with the $5.5 \mathrm{mM}$ PFB solutions used ( $\mathrm{a}$ and $\mathrm{b}$ ). These nanocorrals are denoted as "partially filled nanocorrals" in the detailed statistics (Figure S6), but were not included in the summarized polymorph distribution statistics. With a BFA concentration of $6.6 \mathrm{mM}$ most nanocorrals remained partially filled $(\mathrm{c})$, hence the concentration was increased to $10 \mathrm{mM}$. Imaging parameters: $I_{\mathrm{t}}=70 \mathrm{pA}, V_{b}=$ $-0.700 \mathrm{~V}$. 
S9. Single domains in larger nanocorrals

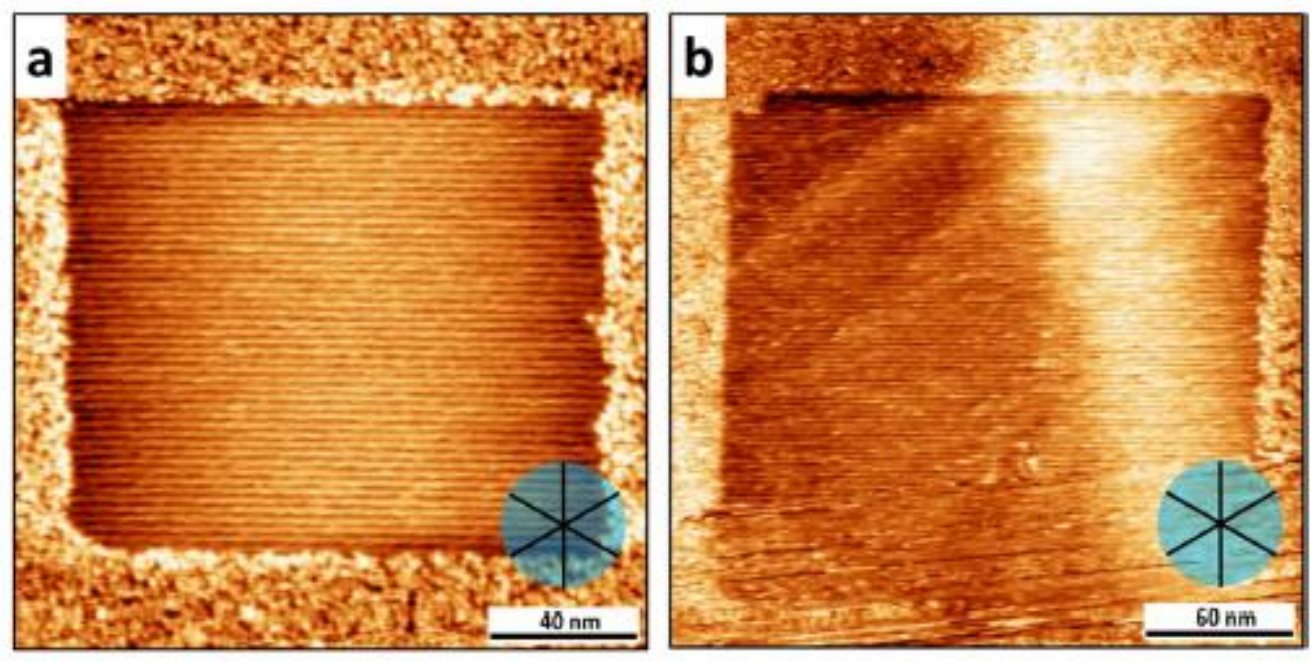

Figure S8. Representative STM images showing a single domain of S-PFB in an approximately $150 \mathrm{~nm} \mathrm{x}$ $150 \mathrm{~nm}(\mathrm{a})$ and $220 \mathrm{~nm} \times 220 \mathrm{~nm}$ (b) $+30^{\circ}$ nanocorral. These domain areas are larger compared to the ones typically observed upon dropcasting on bare HOPG (Figure S2), indicating that after nucleation, the domain growth during the nanoshaving process is favored over additional nucleation events. Imaging parameters: $I_{t}=70 \mathrm{pA}, V_{b}=-0.700 \mathrm{~V}$. 


\section{S10. Ex-situ nanocorrals}

For ex-situ nanocorrals, first the orientation of the HOPG lattice was determined by scanning the dry sample $\left(\mathrm{a}, I_{\mathrm{t}}=1.0 \mathrm{nA}, V_{b}=-0.001 \mathrm{~V}\right)$. After nanoshaving was performed on the dry sample, the solution containing the molecules was dropcasted onto the sample and subsequently the molecular selfassembly in the nanocorrals imaged $\left(b, l_{t}=70 \mathrm{pA}, V_{b}=-0.700 \mathrm{~V}\right)$.
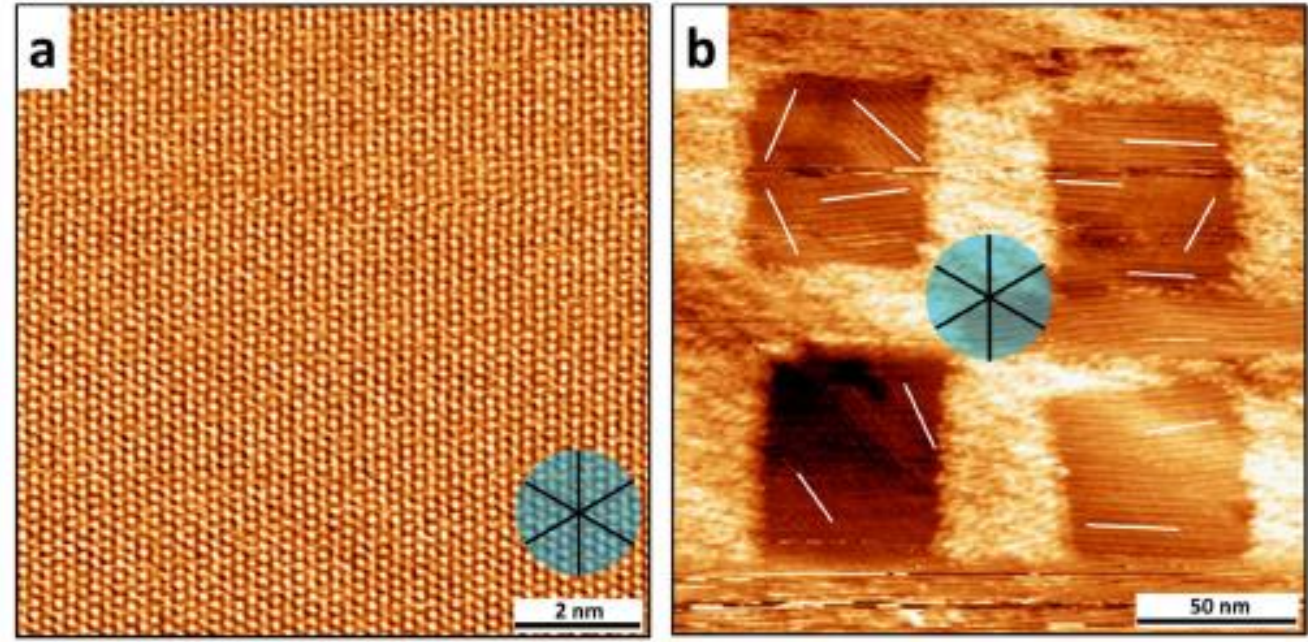

Figure S9. Representative STM image showing the HOPG lattice used to determine the lattice orientation prior to the ex-situ nanoshaving (a) and S-PFB in the ex-situ created nanocorrals (b). In exsitu nanocorrals, multiple domains were frequently observed without a clear bias towards a polymorph or even rotational domains. The orientation of the row-structure in the nanocorrals is indicated by a white line. 
S11. Molecular assembly of BFA in $90^{\circ}$ rotated nanocorrals
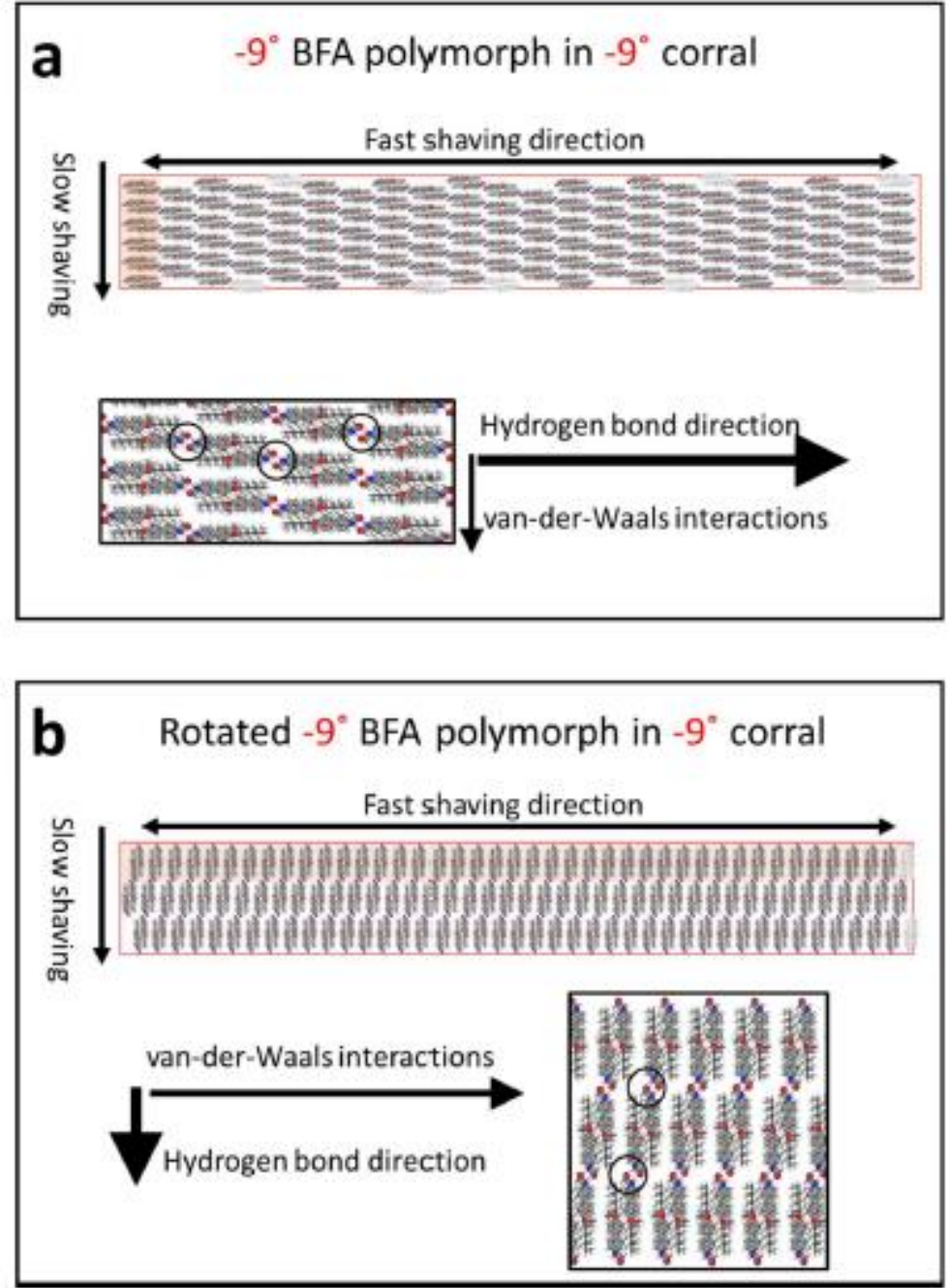

Figure S10. Tentative model showing the molecular alignment of the preferentially formed BFA polymorph in the corresponding corral and the directionality of the intermolecular interactions (a). The not observed, hypothetical alignment of the same polymorph rotated by $90^{\circ}$ is shown in b. In this case, the row structure would be aligned along the fast shaving direction and thus the intermolecular vdW interactions, while H-bonds would be formed along the slow shaving direction. Further, while the number of molecular dimers is higher in the not observed orientation shown in b (119 vs 136), the number of potentially formed $\mathrm{H}$-bonds is lower (107 vs 90 ). The alignment in b corresponds to the nanocorrals shown in figure $\mathrm{S} 5 \mathrm{C}$. 
S12. Molecular assembly of PFB in $90^{\circ}$ rotated nanocorrals
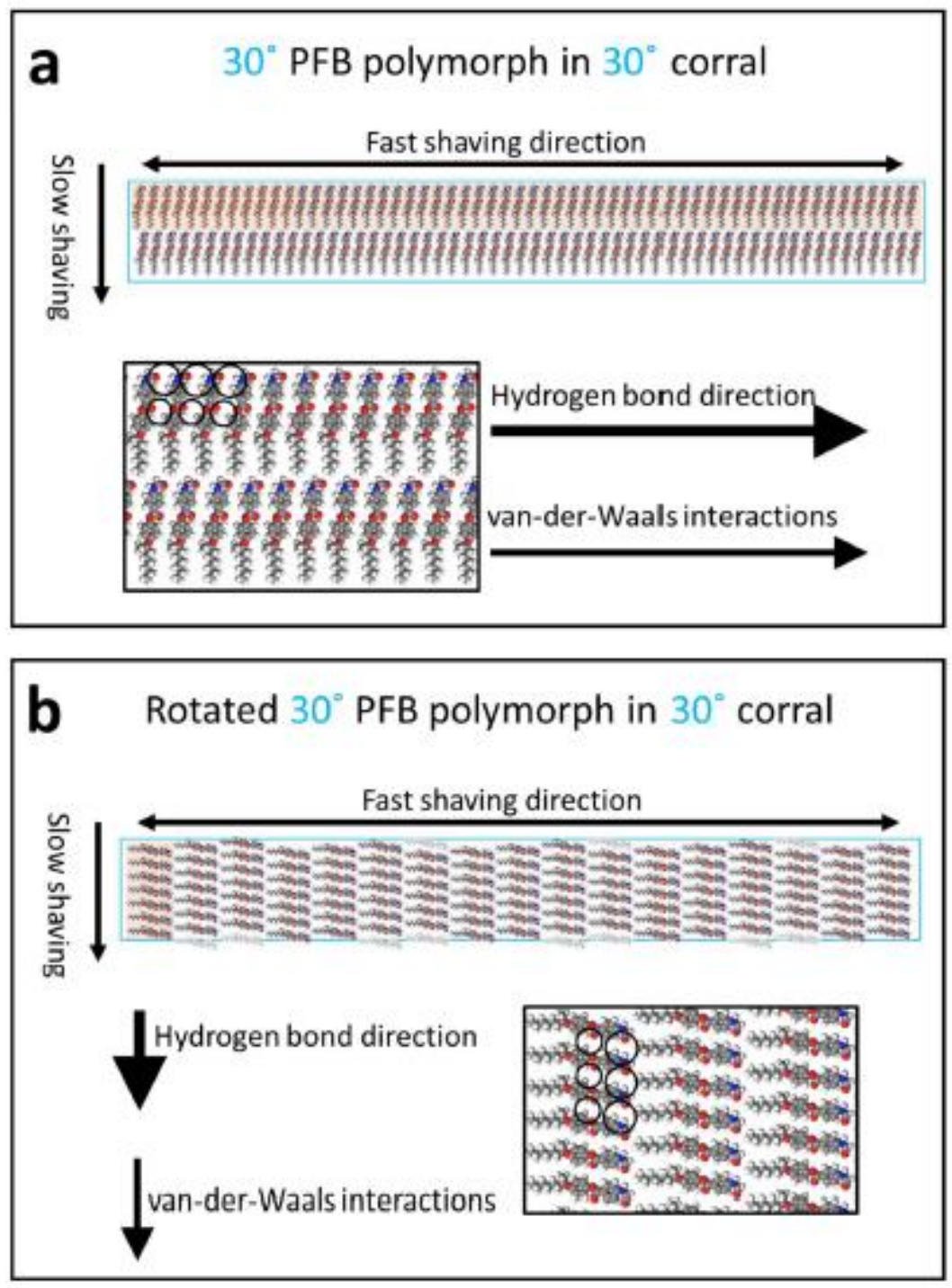

Figure S11. Tentative model showing the molecular alignment of the preferentially formed PFB polymorph in the corresponding corral and the directionality of the intermolecular interactions (a). The not observed, hypothetical alignment of the same polymorph rotated by $90^{\circ}$ is shown in b. In this case, the row structure would be aligned along the slow shaving direction and thus the intermolecular vdW interactions and $\mathrm{H}$-bonds would be formed along the slow shaving direction. Further, while the number of molecules in both cases is very similar (116 vs 119), the number of potentially formed H-bonds (114 vs 102) is lower in the not observed orientation shown in b. The alignment in b corresponds to the nanocorrals shown in figure $\$ 5 \mathrm{a}$ and $\mathrm{b}$. 


\section{S13. Narrow rectangular nanocorrals}
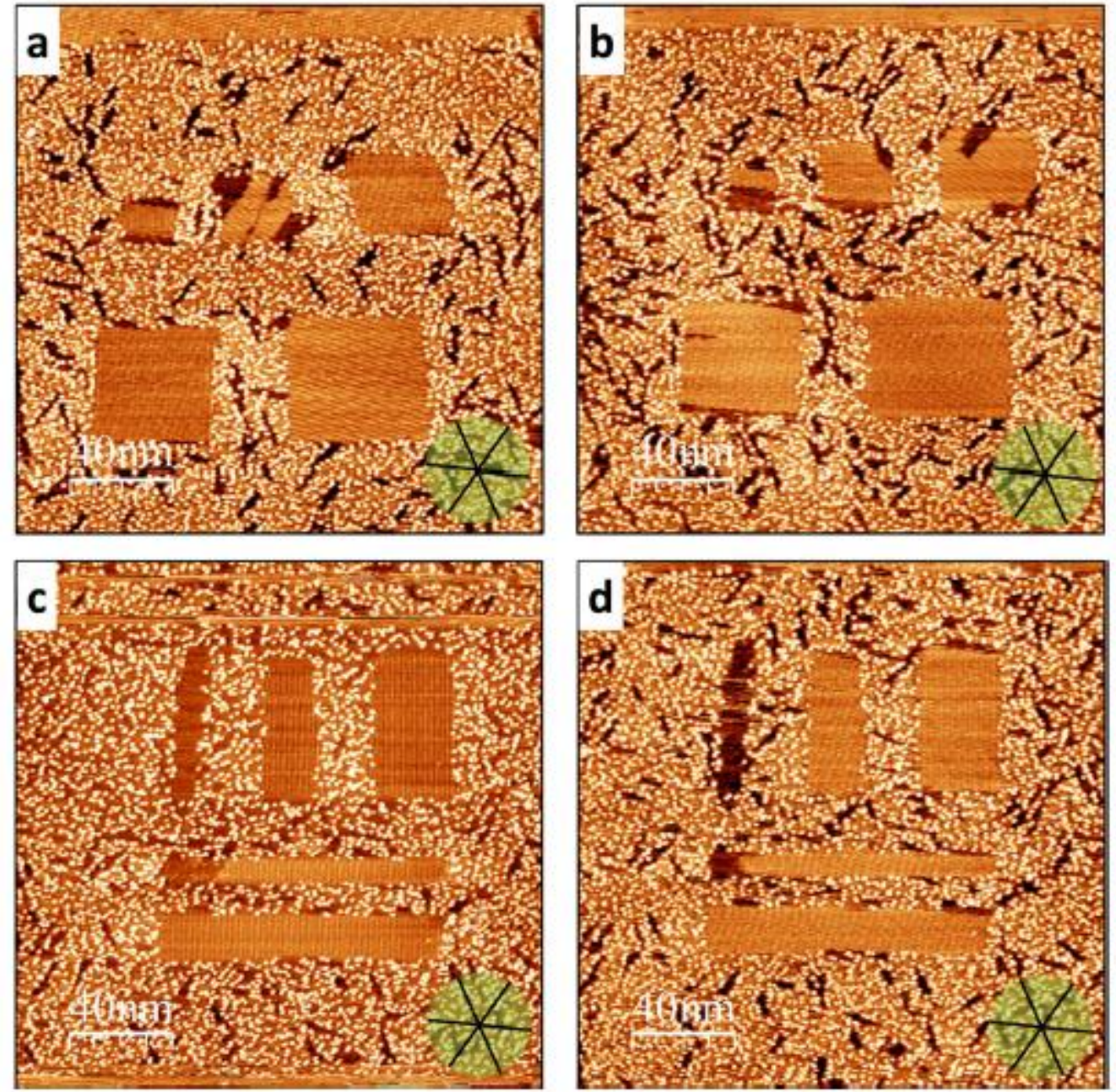

Figure S12. STM images showing the self-assembly of R-BFA in smaller nanocorrals, showing the appearance of partially filled nanocorrals with decreasing size (a and b). In narrow rectangles with the long axis of the rectangles perpendicular as well as parallel to the fast shaving direction self-assembly of $R$-BFA in $\sim 10 \mathrm{~nm}$ high rectangles is only observed when the long axis of the rectangle is parallel to the fast shaving and thus $\mathrm{H}$-bonding direction, while $\sim 10 \mathrm{~nm}$ wide rectangles remain empty, thus indicating anisotropic critical nucleation dimensions. Imaging parameters: $I_{t}=70 \mathrm{pA}, V_{0}=-0.700 \mathrm{~V}$. 
S14. Polymorph distribution after annealing

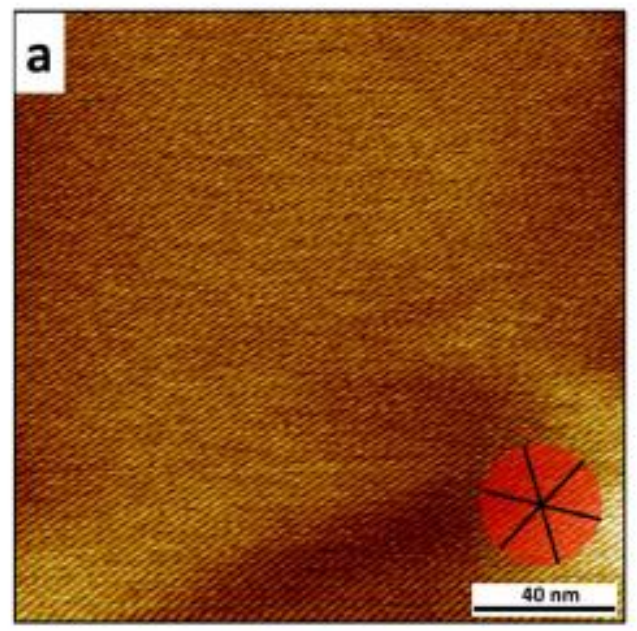

\begin{tabular}{|c|c|c|}
\hline Nr. of images & Area \pm 9 polymorph & Area \pm 24 polymorph \\
\hline 26 & $840000 \mathrm{~nm}^{2}$ & $200000 \mathrm{~nm}^{2}$ \\
\hline & $81 \%$ & $19 \%$ \\
\hline
\end{tabular}

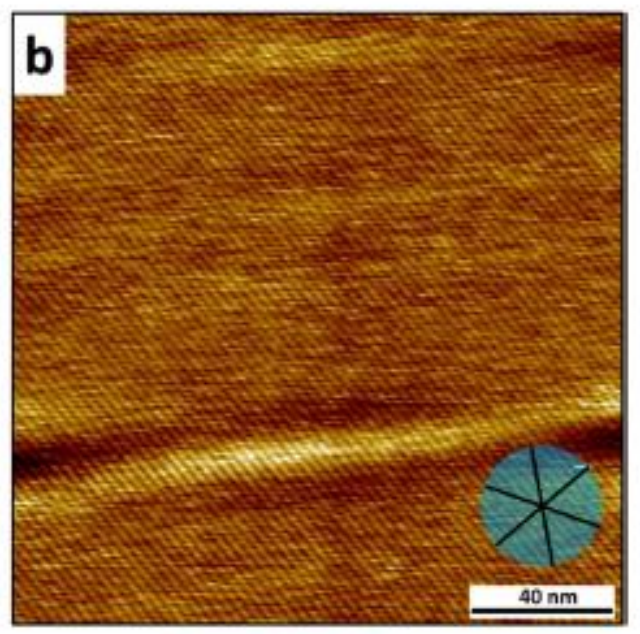

\begin{tabular}{|c|c|c|}
\hline Nr. of images & Area \pm 22 polymorph & Area \pm 30 polymorph \\
\hline 27 & $40000 \mathrm{~nm}^{2}$ & $1040000 \mathrm{~nm}^{2}$ \\
\hline & $4 \%$ & $96 \%$ \\
\hline
\end{tabular}

Figure S13. Representative STM images of R-BFA (a) and S-PFB (b) self-assembly on pristine HOPG after annealing the sample at $80^{\circ} \mathrm{C}$ for five minutes. For both molecules, the annealing lead to single domains exceeding $200 \mathrm{~nm} \times 200 \mathrm{~nm}$ in size. To avoid measuring the same domain several times, five $200 \mathrm{~nm} \times$ $200 \mathrm{~nm}$ STM images were taken within the available scan area of approximately $1.2 \mu \mathrm{m} \times 1.2 \mu \mathrm{m}$ (one in each corner and one in the center). Afterwards, the sample was moved several micrometers to scan a different location. Compared to the polymorph distribution at room temperature (Figure S2), the relative appearance of the minority polymorph further decreases for both systems, as shown in the statistics below the images. Imaging parameters: $I_{t}=70 \mathrm{pA}, V_{0}=-0.700 \mathrm{~V}$. 